\title{
FISSION CANDIDATES AMONG DETACHED CLOSE BINARIES*
}

\author{
H.-A. OTT \\ Astronomisches Institut der Universität Münster, F.R.G.
}

(Received 26 July, 1983)

\begin{abstract}
A comparison between results of model calculations and observed properties of close, but detached low mass binaries with Main Sequence primaries shows statistical trends, which seem to support fission origin for some of these objects: the mass-momentum relation, the relation between mass ratio and separation and the relation between mass ratio and synchronisation speak in favour of close initial separations and small mass ratios of the components.
\end{abstract}

\section{Introduction}

Fission, i.e. splitting of a dynamically collapsed object (pre-Main Sequence star) by rotational instability, is often proposed as a mechanism of forming close binary systems (CBS), see e.g. Lucy (1981) and other articles in IAU Symp. 93 (1981). Though the theory of rotating polytropes provides us with a rough criterion for instability, that can be applied to models of low mass protostars (Roxburgh, 1966), the physics of the fission process itself and the characteristic properties remain largely unknown. The rationale of this work is to estimate these properties.

Two basic assumptions are: (1) fission is a process which occurs in single rotating objects not able to store large amounts of momentum; (2) fission forms two gravitationally bound stars which star their dynamical evolution from a very small initial separation.

\section{A Survey of the Literature}

Some theoretical results and statistical analyses of CBS observations constitute the background for these studies and are summarized in Table I. Throughout this work we use the following units and definitions:

Mass, distance, time in solar units: solar mass, astronomical unit, year.

Momentum units can easily be derived.

Separation: in units of sum of radii of the two components.

Mass ratio: = mass (secondary)/mass (primary).

Synchronisation: = orbital frequency/rotational frequency (primary).

Assuming that Roxburgh's theory gives the correct momentum $H_{0}(M)$ for stellar masses smaller than 5 , we have to define parametrically the status of a close binary

* Paper presented at the Lembang-Bamberg IAU Colloquium No. 80 on 'Double Stars: Physical Properties and Generio Relations', held at Bandung, Indonesia, 3-7 June, 1983. 


\section{TABLE I}

Fission - parameters in theory and observation

SB 1 - single line spectroscopic binary

SB 2 - double line spectroscopic binary

\begin{tabular}{|c|c|c|}
\hline Total mass $(M)$ & Mass ratio $(Q)$ & $\begin{array}{l}\text { Momentum }(H) \text {, separation }(A) \\
\text { period }(P)\end{array}$ \\
\hline \multicolumn{3}{|l|}{ Theory: } \\
\hline $\begin{array}{l}\text { Roxburgh (1966): } \\
\text { pre-Main Sequence } \\
\text { stars (convective- } \\
\text { radiative phase): }\end{array}$ & $\begin{array}{l}\text { Lucy }(1981) \text { : } \\
N \text {-particle simulations } \\
\text { favour small } Q(0.2-0.3)\end{array}$ & $\begin{array}{l}\text { Roxburgh (1966): } \\
H=H_{0}(M) \text { computed } \\
\text { for mass range } 0.8 \leqq M \leqq 5\end{array}$ \\
\hline $\begin{array}{l}\text { Fission requires } \\
M \geqq 0.8 \\
M>4 \text { result in } \\
\text { detached systems }\end{array}$ & $\begin{array}{l}\text { Generally accepted: } \\
Q \ll 1 \text { (no serious } \\
\text { theoretical foundation) }\end{array}$ & $\begin{array}{l}\text { Van 't Veer }(1979) \text { : strong } H \text { loss in } \\
\text { contact systems results in } \\
\text { evolution to smaller } Q \text {. Form all } \\
\text { contact systems with } Q \text { near } 1 \text { ? }\end{array}$ \\
\hline \multicolumn{3}{|l|}{ Observation: } \\
\hline $\begin{array}{l}\text { Staniucha (1979): } \\
\text { statistical analysis } \\
\text { of the catalogue by } \\
\text { Batten } \text { et al. }(1978) \text { : } \\
{ }^{*} \text { selection effects } \\
\text { strong for } M<1 \\
{ }^{*} \text { peak of distribution } \\
\text { near } M=1.2 \\
\text { for SB } 2\end{array}$ & $\begin{array}{l}\text { Staniucha }(1979) \text { : } \\
Q \text { distribution bimodal } \\
\text { (peak near } 0.2 \text { for SB } 1 \\
\text { and } 1 \text { for SB 2) } \\
\text { Trimble (1978): } \\
\text { non-evolved systems } \\
\text { show the peak near } 0.2 \\
\text { (i.e. no evolution } \\
\text { effect) }\end{array}$ & $\begin{array}{l}\text { Staniucha (1979): } \\
\text { distributions of } P \\
\text { (peak near } 1.5 \text { days) and } \\
\text { Asini (peak near } 17 R_{\odot} \text { ) strongly } \\
\text { biased, no significance for star } \\
\text { formation } \\
\text { Mochnacki (1981): } \\
\text { contact systems (WUMa) } \\
\text { often show too much } H / M \\
\text { (theory incorrect?) }\end{array}$ \\
\hline \multicolumn{2}{|c|}{$\begin{array}{l}B(V) \text { stars show bimodal distribution } \\
\text { (peak near } M=7 \text { and rise } \\
\text { for } M<4 \text { ) }\end{array}$} & $\begin{array}{l}\text { Farinella et al. (1979): } \\
\text { from late } B \text { to } G \text { stars: } \\
\text { common average geometry, } \\
\text { separation about } 3 \\
\text { (sum of radii of stars) }\end{array}$ \\
\hline
\end{tabular}

after the (hypothetical) fission. Hints from the literature and simple momentum considerations lead to the following plausible scenario: the phase shortly after fission is characterized by a low separation $(A<3)$ and a small mass ratio $(Q<0.3)$ together with a rotational frequency of the primary, which is not much higher than the orbital frequency of the companion $(S>0.2)$. Possible mass and momentum loss during the fission process is not included in our preliminary computations.

\section{Orbital Evolution in Detached Close Binaries}

An answer to the question which observed CBS are likely to be fission candidates, is only possible, if the dynamical evolution of the systems can be traced over a long time interval, e.g. the main sequence lifetime of the primary. In order to understand some statistical trends in our sample of observed CBS (see next section), we here 
present some results of model calculations concerning the evolution of separation and synchronisation with time.

The dynamical equations for tidal dissipation in stars with convective envelopes (Zahn, 1977) have been integrated numerically under the assumptions of: (i) insignificant mass loss or transfer; (ii) circular orbits; (iii) possible steady momentum loss of the primary (magnetic braking of a stellar wind). Fixed parameters are: total momentum $H=H_{0}(M)$ from Roxburgh and initial separation $A=1$. Depending on the chosen total mass $(M)$ and mass ratio $(Q)$ the calculations trace the orbital evolution with or without momentum loss. The upper time limit is the main sequence lifetime of the primary $\left(6 \times 10^{9} \mathrm{M}^{-2}\right)$.

Figure 1 shows the final separation as a function of model mass and mass ratio, all without momentum loss. Figure 2 shows the final synchronisation for the same models as in Figure 1. The curves represent upper limits for empirical CBS, because the ages of observed systems with main sequence primaries fall well within the given evolutionary limit.

The results of calculations including momentum loss are more complex and difficult to interpret. In general they show smaller final separations, as expected, but the distance between the components can reach a maximum, before it slowly approaches a final value. Synchronisation sometimes reaches unity very early, but goes through prolonged oscillatory phases afterwards - orbital evolution continues!

We will not discuss these results here, because presently available empirical data

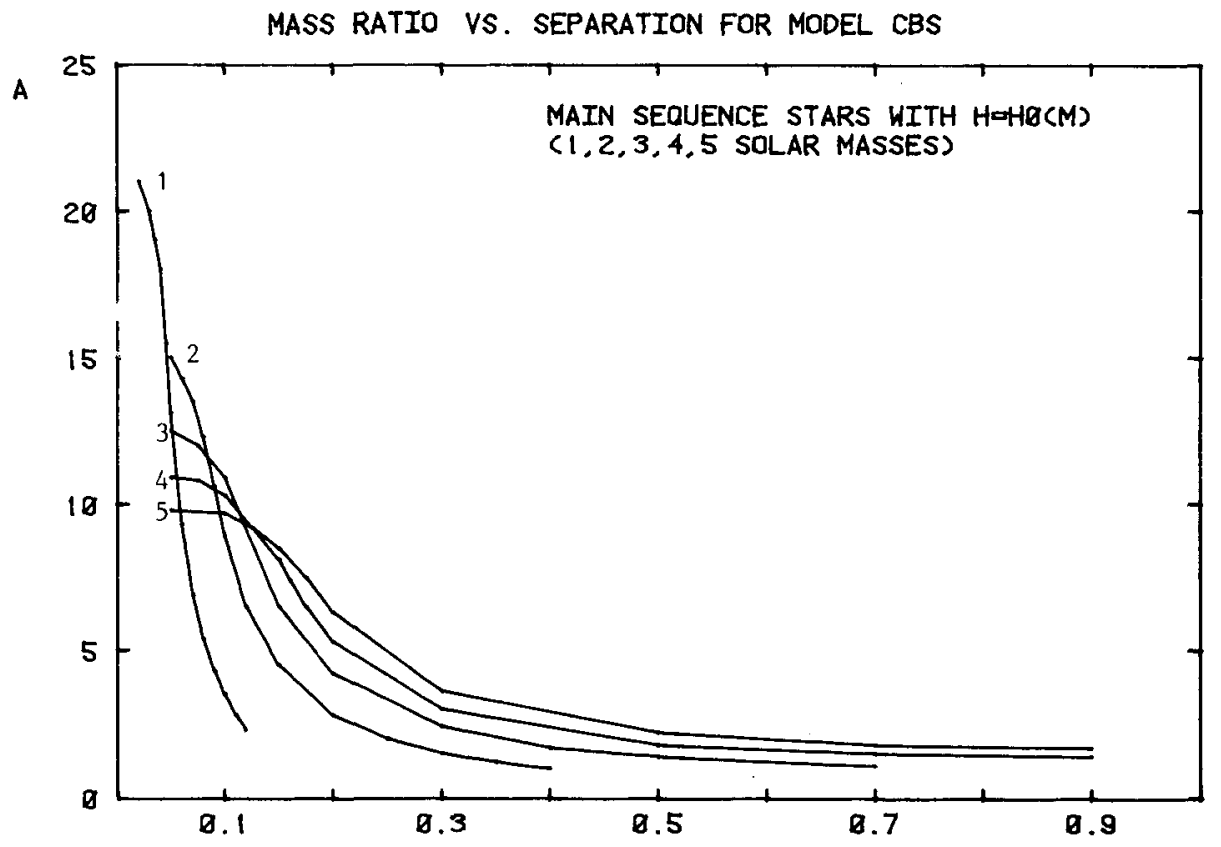

Fig. 1. System configuration with respect to separation $(A)$ after one Main Sequence lifetime of the primary. 
MASS RATIO VS. SYNCHRONISATION FOR MODEL CBS

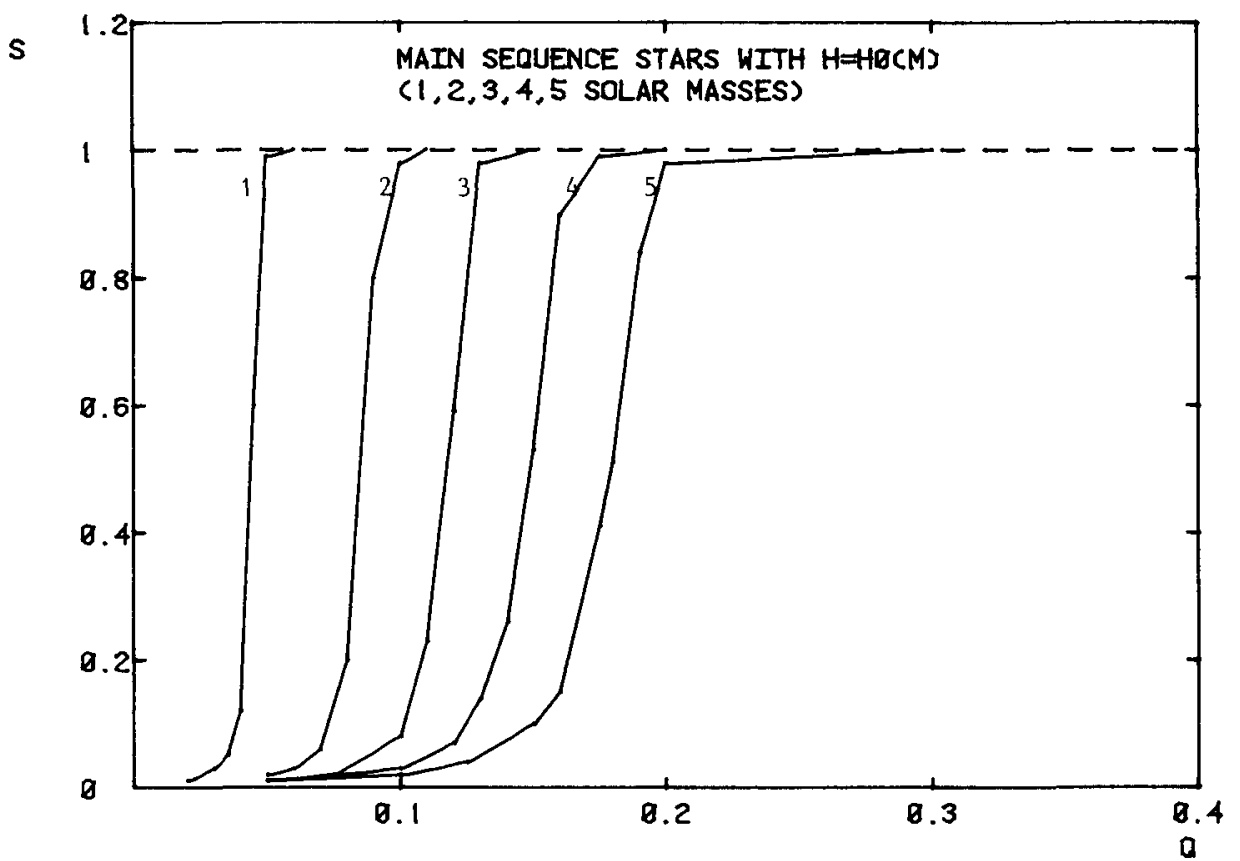

Fig. 2. System configuration with respect to synchronisation $(S)$ after one Main Sequence lifetime of the primary.

provide arguments in favour of models without momentum loss. For the future more and more refined model computations are planned. Comparison of the present models with the empirical data will follow in the next section.

\section{Observed CBS: Fission Candidates?}

We propose two basic empirical indicators in order to distinguish statistically between fission candidates and other stars: (1) the total momentum should have values around $H_{0}(M)$ according to Roxburgh; (2) the synchronisation should be well developed (not too far from unity), as expected from the small initial separation.

Our analysis is confined to single line detached systems with known rotation of the primaries (about 70 systems). The orbital elements are from the catalogue of Batten et al. (1978), the rotational data from the catalogue of Bernacca and Perinotto (1970). This sample is neither complete nor unbiased. The chosen indicators however should be applicable to this inhomogenous sample, an assumption which will be tested by Monte-Carlo simulations. 


\subsection{Mass-momentum Relation $(M / H)$}

According to Brosche (1963) a universal relation exists between momentum and mass $\left(H \sim M^{2}\right)$, for double stars with a larger internal scatter than for single stars on the main sequence. If this is correct, fission objects - formed from single stars -should show a better defined $M / H$ relation than systems with components of independent origin.

Taking synchronisation as fission indicator, this expectation is confirmed by Figures 3 and 4 . Most of the data points lie within reasonable limits around Roxburgh's results, for small values of $Q(0$ to 0.3$)$ the $M / H$ relation is better defined than for larger $Q$ values, Systems with known inclination angles $i$ display the relation even better.

\subsection{Mass ratio and Separation $(Q / A)$}

Our model calculations show a clear correlation between mass ratio and final separation for Main Sequence stars with Roxburgh's momentum (Figure 1). The rapid stellar evolution of the more massive primaries does not allow the separation to reach the synchronous stage during the Main Sequence lifetime. This trend has a good empirical counterpart in the diagram of Figure 6 (synchronous systems with momentum near $H_{0}(M)$ ), but non-synchronous CBS or such with excess

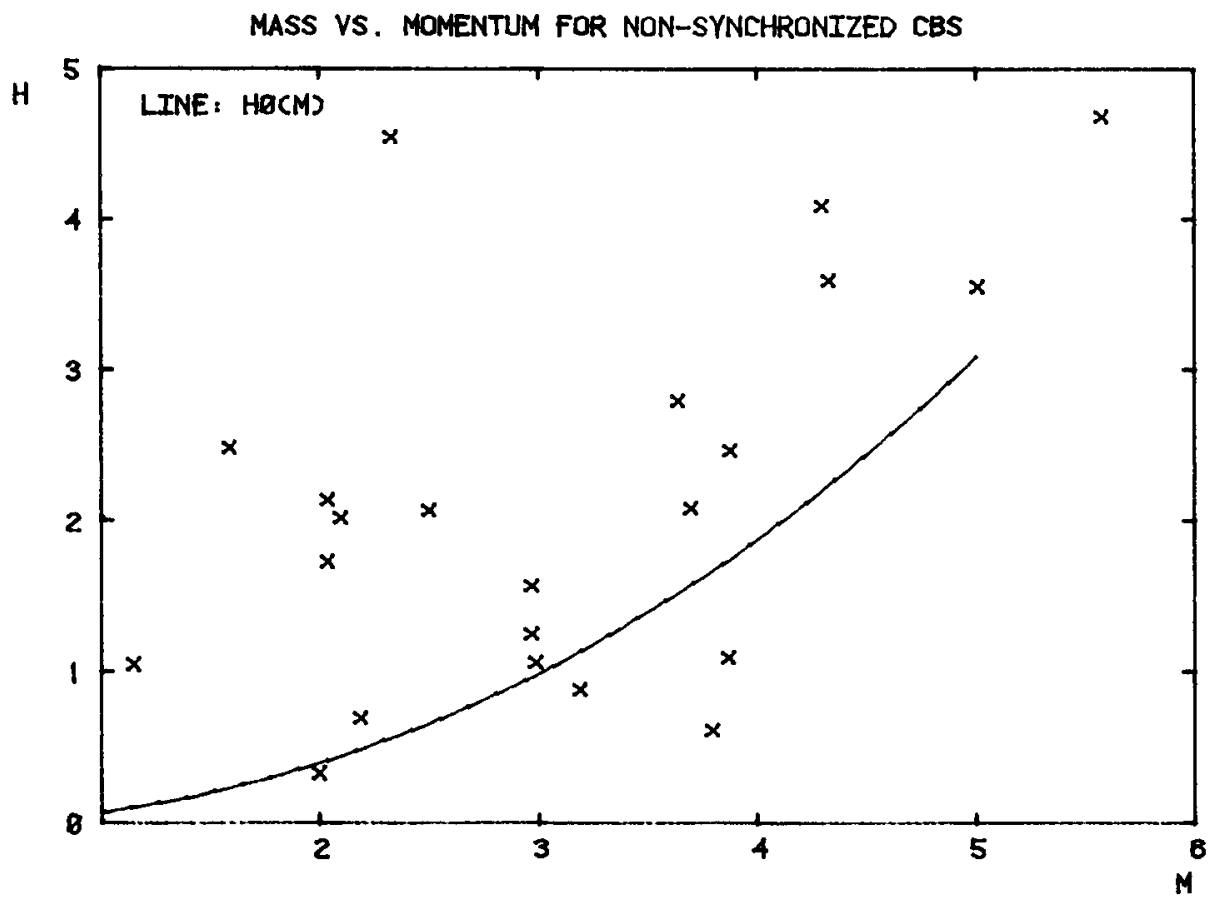

Fig. 3. Observed mass-momentum relation for non-synchronized CBS. Line represents Roxburgh's theoretical relation for fission objects. 


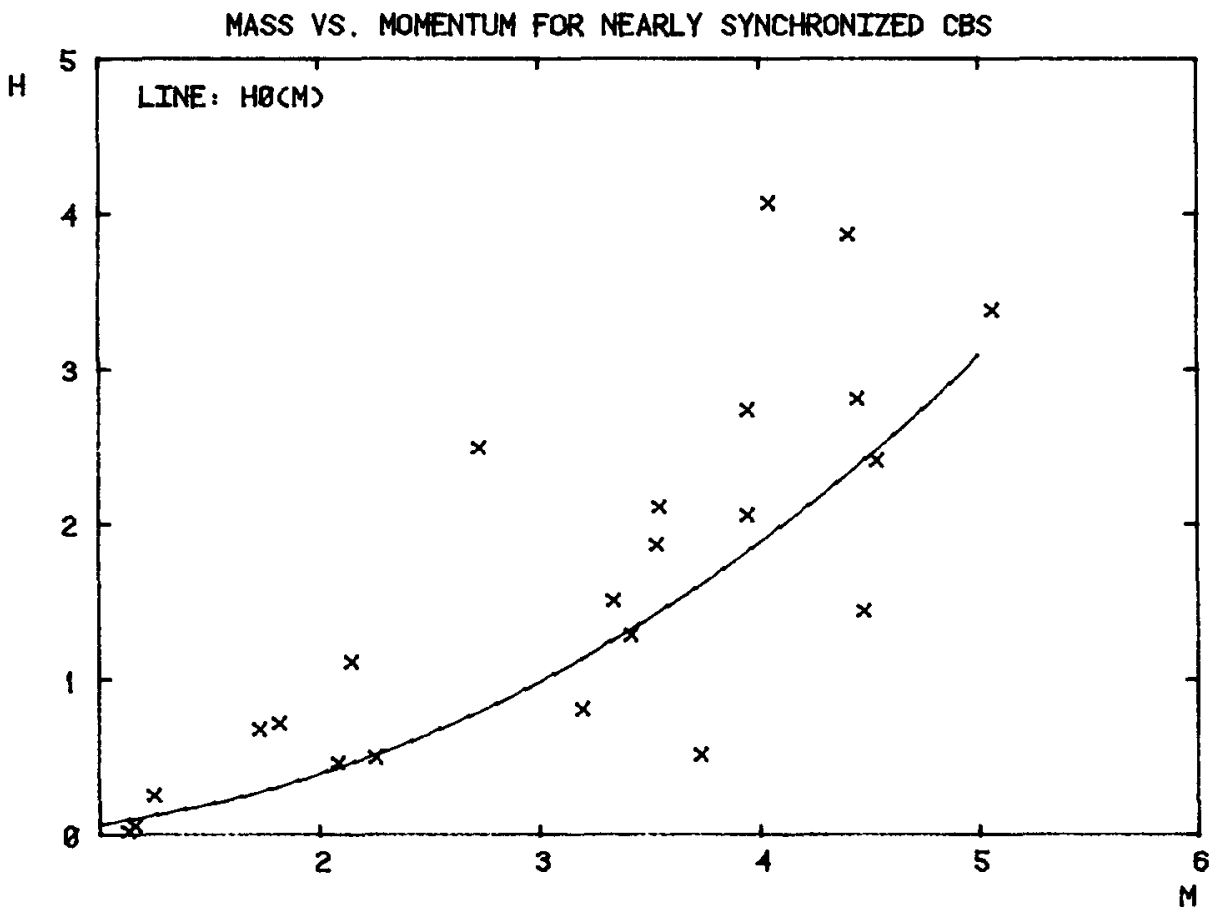

Fig. 4. Observed mass-momentum relation for nearly synchronized CBS. Line represents Roxburgh's theoretical relation for fission objects.

MASS RATIO VS, SEPARATION FOR CBS

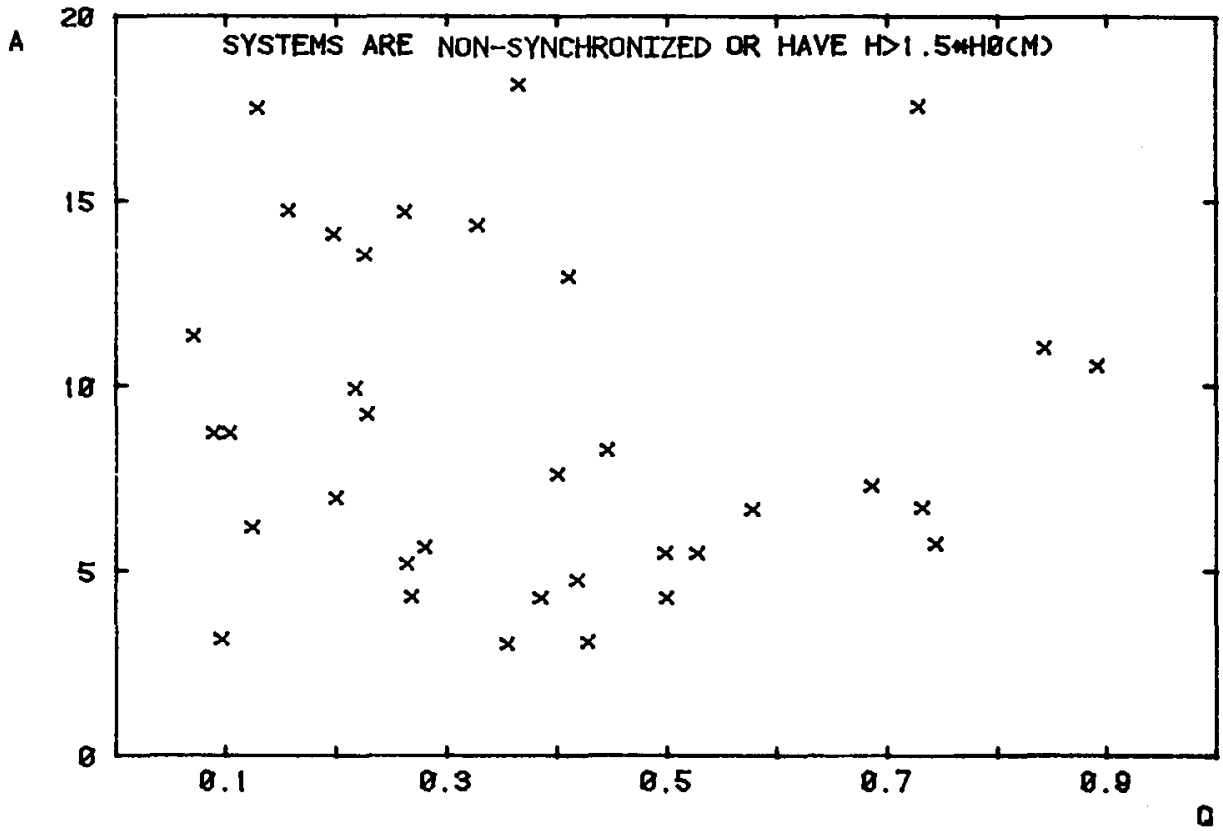

Fig. 5. Observed combinations of system parameters for non-synchronized or high momentum CBS. Compare with model results of Figure 1. 


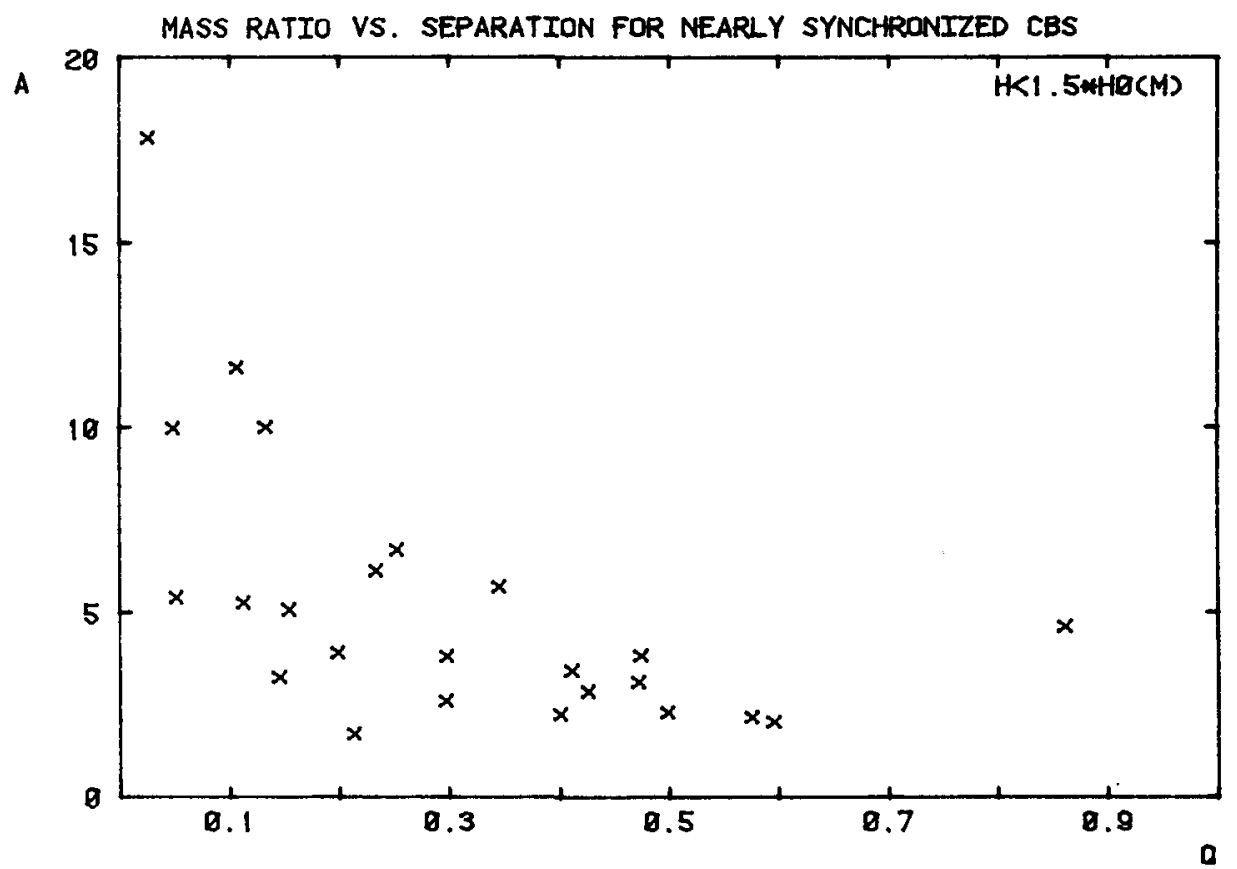

Fig. 6. Observed combinations of system parameters for nearly synchronized and low momentum CBS. Compare with model results of Figure 1.

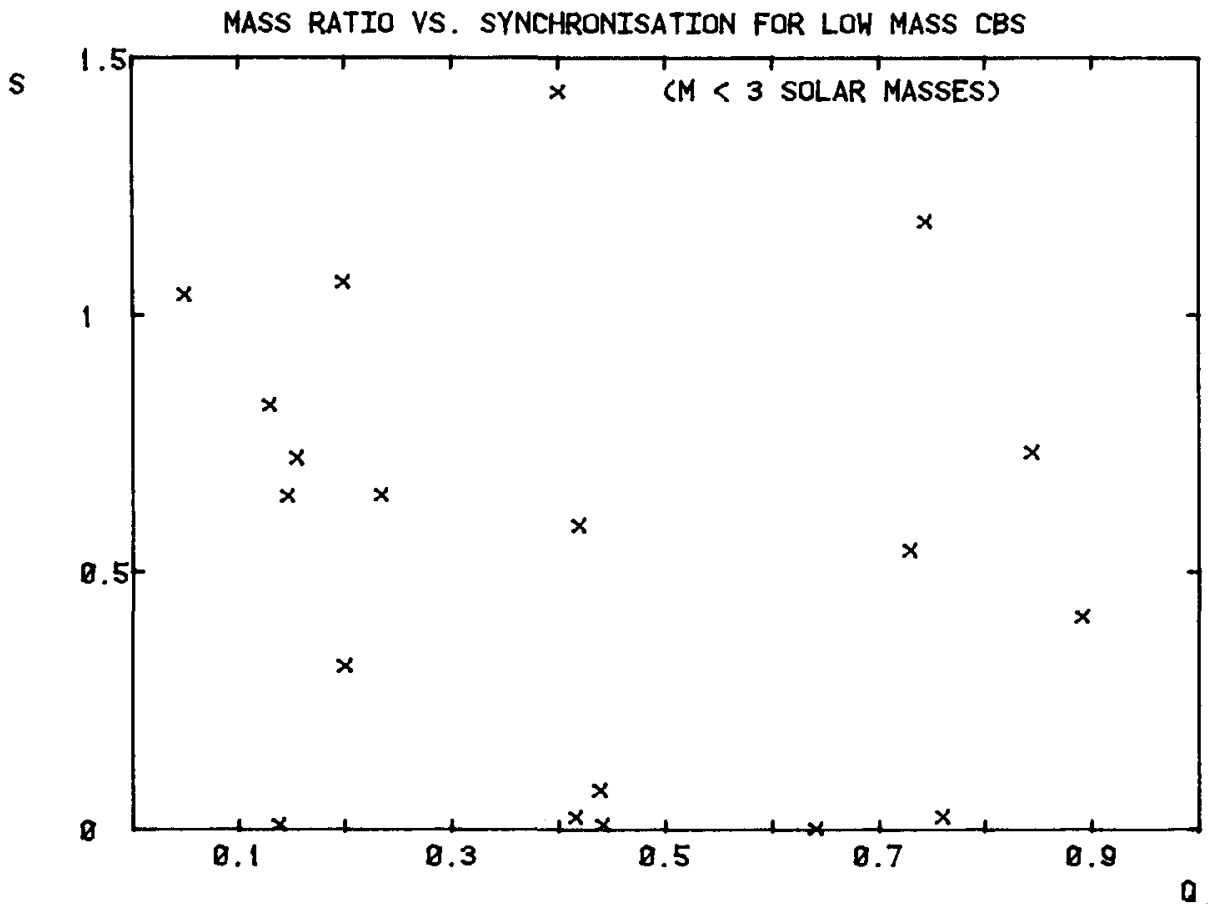

Fig. 7. Observed combinations of mass ratio and synchronisation for low mass CBS. All evolved fission candidates should have reached synchronisation, viz. Figure 2. 
MASS RATIO VS. SYNCHRONISATION FOR HIGH MASS CBS

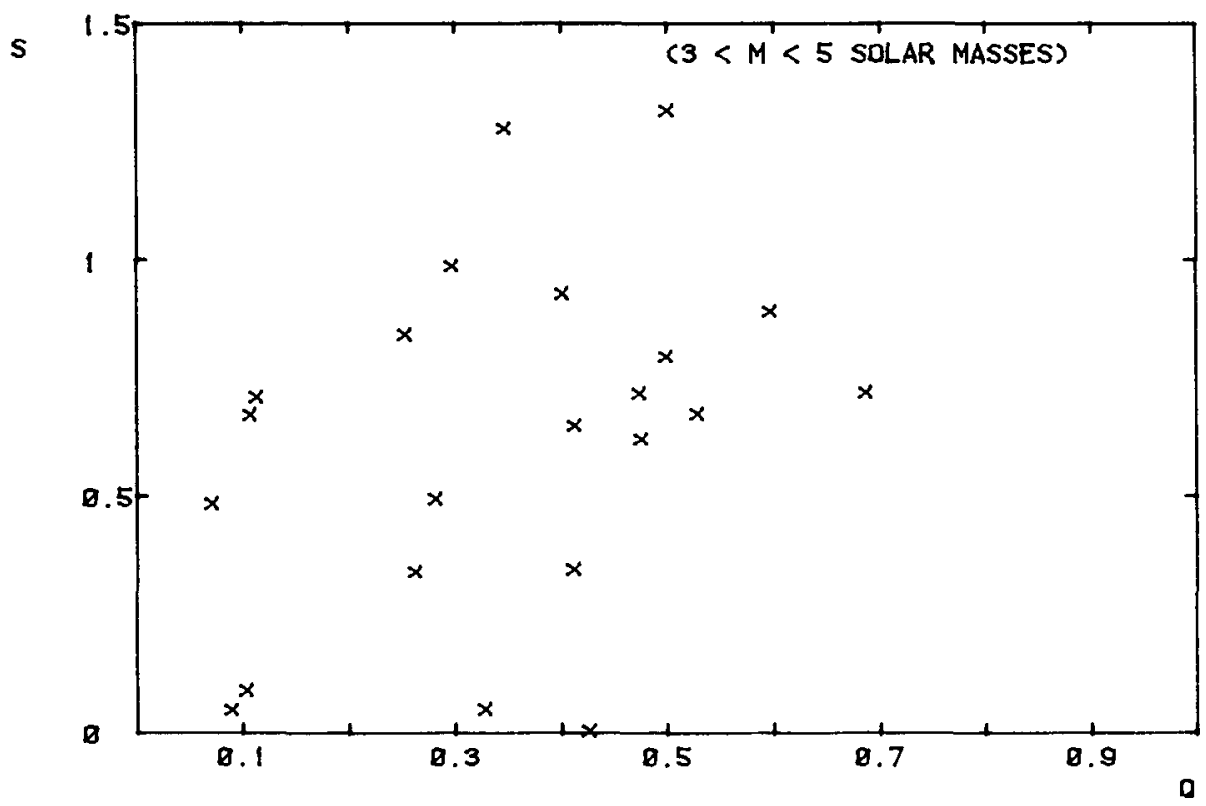

Fig. 8. Observed combinations of mass ratio and synchronisation for high mass CBS. Only evolved fission candidates with large $Q$ values should have reached synchronisation, viz. Figure 2.

momentum do not show this behaviour (Figure 5). These observational results support the assumption of small initial separations among synchronous CBS.

\subsection{MASS RATIO AND SYNCHRONiSATION $(Q / S)$}

Model calculations show (see Figure 2), that Main Sequence primaries of CBS with masses from 1 to 5 and Roxburgh's momentum do not always rotate synchronously, even if they started near contact separation; large masses (3 to 5 ) and small $Q$ values $(<0.2)$ lead to nonsynchronous systems. The empirical tests of this expectation are presented in Figures 7 and 8 . Because of the small sample size only two mass ranges are considered. Nevertheless the trend seems clear: the more massive systems populate the area 'small $Q$ /nearly synchronous state' in Figure 8 to a lesser degree than the low mass systems of Figure 7.

If this trend can be confirmed statistically, we find a possibility to separate nonfission candidates from other stars: more massive systems with small $Q$ and Roxburgh's momentum, which appear synchronous, cannot have started their orbital evolution from a close initial geometry.

Another interesting fact is that all (11) low mass systems with $Q>0.25$ possess too much momentum to be fission candidates, in contrast to the more massive systems, among which only 7 out of $17(41 \%)$ have larger momentum values. This 
and the above remark on the $M / H$ relation, concerning $Q$, speak in favour of small $Q$ values to be characteristic of fission candidates.

\section{Summary}

Our comparison between results of model calculations without momentum loss and observed properties of detached low mass CBS with main sequence primaries shows some statistical trends, which seem to support fission origin for some of these objects. If Roxburgh predicts the correct momentum values for fission of protostars, the mass-momentum relation, the relation between mass ratio and separation and the relation between mass ratio and synchronisation speak in favour of: (1) a very close initial separation of fission candidates; and (2) small mass ratios of the binary components.

Of course, more detailed models and further statistical confirmation are needed to strengthen the argument for fission origin of some close binary stars.

\section{References}

Batten, A. H., Fletcher, J. M., and Mann, P. J.: 1978, Publ. Dominion Astrophys. Obs. 15, No. 5

Bernacca, P. L. and Perinotto, M.: 1970, Contr. Oss. Astrofiz. Padova, No. 239.

Brosche, P.: 1963, Z. Astrophys. 57, 143.

Cassinelli, J. P.: 1979, Ann. Rev. Astron. Astrophys. 17, 275.

De Grève, J. P. and Vanbeveren, D.: 1980, Astrophys. Space. Sci. 68, 433.

Farinella, P., Luzny, F., Mantegazza, L., and Paolicchi, P.: 1979, Astrophys. J. 234, 973.

Lucy, L. B. : 1981, in D. Sugimoto, D. Q. Lamb, and D. N. Schramm (eds.), 'Fundamental Problems in the Theory of Stellar Evolution', IAU Symp. 93, 75.

Mochnacki, S. W.: 1981, Astrophys. J. 245, 650.

Roxburgh, I. W.: 1966, Astrophys. J. 143, 111.

Staniucha, M.: 1979, Acta Astron. 29, No. 4, 587.

Trimble, V.: 1978, Observatory, 98, 163.

Van 't Veer, F.: 1979, Astron. Astrophys. 80, 287.

Zahn, J.-P.: 1977, Astron. Astrophys. 57, 383. 